\title{
Beyond personalized medicine
}

\section{"I argue for the necessity of a more holistic view of individualized medicine without equating it to pharmacogenomics."}

As it says in the Old Testament:

\section{"To every thing there is a season, and a time to every purpose under the heaven."}

Andy Warhol was more cynical in 1968 when he said that, in the future, everyone would be world famous for $15 \mathrm{~min}$. Nonetheless, pharmacogenetics and later, pharmacogenomics, have now been part of the scientific community for more than 50 years. The past decade has brought new vitality and popularity to pharmacogenetics, primarily as a result of the knowledge gained from mapping the human genome. A simple search on Pubmed using 'pharmacogenetics' as the search word reveals approximately 6200 articles out of a total of 7600 articles dealing with aspects of pharmacogenetics were written in the past 10 years, and approximately 3500 of them were published in the past 5 years [101]. In addition, knowledge concerning pharmacogenomics is no longer confined to clinical pharmacological research. Specialists, general practitioners, pharmacists and other healthcare professionals have been making acquaintance with pharmacogenomics. Pharmacogenetics and pharmacogenomics are making their way into the curriculum at a number of universities and there is a great deal of support for reinforcing teaching on the subject. Even the general public show some degree of knowledge regarding the potential of pharmacogenetics. Since there are no signs that pharmacogenomics is on its way out of the spotlight, perhaps it should be seen in the correct light.

Attitudes towards technologies appear to undergo a cyclical process. The psychologist Max Seige called this process 'Seige's cycle' in 1912. The first phase of this cyclical process is characterized by hope, opportunity and euphoria. The second phase is marked by scepticism and criticism, and the third by disappointment and the search for better alternatives and new hope [1]. The aim of this article is to illustrate the path of pharmacogenomics through Seige's cycle, from euphoria to disappointment. On this basis, I argue for the necessity of a more holistic view of individualized medicine without equating it to pharmacogenomics. The holistic approach offers new hope for individualized medicine being the solution to the strategy to put the right pill in the right mouth.

\section{Pharmacogenetics: hype \& thrust}

The past decade has seen increased international focus on the research field of pharmacogenetics and pharmacogenomics with the aim of developing and using drugs based on individual patient genotypes $[2,3]$. In its purest form, pharmacogenetics and pharmacogenomics have ignited expectations of major therapeutic advances that will lead to the development of more specific therapeutic regimes with better efficacy and fewer side effects.

A short review of the scientific literature dealing with pharmacogenomics attests to the great expectations and belief in technological progress. In 2004, the Massachusetts Institute of Technology Review (MA, USA) called pharmacogenetics/pharmacogenomics one of the ten most promising technologies. Headlines of articles in scientific journals in the period leading up to 2004 also give the impression of a very positive perception of the opportunities within pharmacogenomics. Below are two examples that are representative of many other headlines:

"From ready-made to tailor-made - future possibilities for individualized drug therapy."

\section{"Pharmacogenomics: unlocking the human genome for better drug therapy."}

Scientific colleagues who have been following the discussion will nod in recognition at these expectations, and in some cases euphoria,

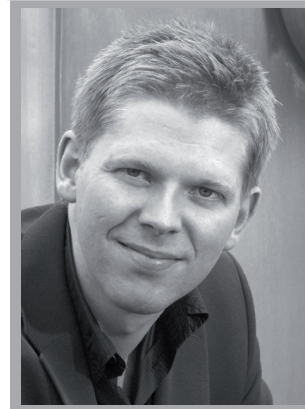

Claus Møldrup ${ }^{\dagger}$

${ }^{+}$Author for correspondence.

University of Copenhagen,

Faculty of Pharmaceutical

Sciences, Department of

Pharmacology and

Pharmacotherapy,

Section for Social Pharmacy,

Universitetsparken 2,

DK-2100 Copenhagen,

Denmark

Tel.: +453533 6452;

Fax: +45 3533 6050;

cm@farma.ku.dk 
evidenced above. I admit that in my early scientific articles and popular science writings on the subject, I too expressed an optimism that has not proved scientifically durable ... yet?

\section{Critique \& skepticism of the pharmacogenetics perspective}

Very few of the expectations have been realized at this point in time. The reasons for this are many and complex, involving scientific, political and economic obstacles. The following quote describes, very accurately, the reality in which pharmacogenomics currently exists [4]:

"...however, pharmacogenomics has made only a few inroads into clinical practice to date.

This review evaluates obstacles that need to be overcome. These include the complexity of mechanisms underlying drug response, given singly or in combination, uncertainty about the genetic underpinnings of complex diseases, such as cancer, diabetes, cardiovascular and mental disorders and a lack of quantitative understanding of the scope of genetic variations, even for well-studied genes".

In addition to the influence of these genetic and individual factors on the opportunities to expand pharmacogenomics, it is also worthwhile mentioning that situational factors, such as a lack of education in healthcare professionals as well as patients (including a lack of compliance), contribute further to the difficulty of achieving the maximum from pharmacogenomics. Scepticism regarding the opportunities within pharmacogenetics and pharmacogenomics has been expressed more sharply in recent years. This trend is apparent in scientific articles as well as in conferences concerning pharmacogenetics and pharmacogenomics, where keynote speakers attempt to outdo each other in criticizing the possible perspectives for treatment in the future. According to a critical review by Kollek et al., the clinical value of pharmacogenetics is still not proven [5]. One reason might be that, so far, the approach to pharmacogenetics has been based on the understanding of individualization as a biomechanistic concept, not taking the biopsychosocial aspect into account. This means that the terms 'individualization' or 'personal' refer to a biological pheromone, not taking the individualization of both biological and personal aspects of the individual into account when addressing the individualization of therapies [6,7].

\section{Realism \& alternative approaches to pharmacogenetics}

One obvious consequence of the euphoria surrounding pharmacogenomics is the lack of a holistic perspective regarding personalized medicine. Individualized treatment has been practiced clinically for decades. Pharmacokinetics and pharmacodynamics have been the basis for all pharmacological practice. Regardless, literature attests the fact that pharmacogenomics has slowly become synonymous with personalized medicine. However, the complex character of most diseases and the subsequent complex pharmacotherapeutic regime often make it difficult to achieve optimal benefit using a purely pharmacogenetic and pharmacogenomic strategy. Therefore, it is more meaningful to expand pharmacogenomic access to understanding personalized medicine in the broader sense. This is said being aware that optimal pharmacological treatment is not conditional on the correct genetic conditions alone, but is influenced by many individual and situational factors. In the years ahead, these factors will redefine the generalized 'one-size-fits-all' concept we see today in pharmacotherapy. The future will offer a more holistic view of personalized medicine, which in addition to pharmacogenomic approaches will include:

- Individualized indications arising through a dialogue between doctor and patient;

- Individual choices of drugs that are appropriate to the experience and preferences of both doctor and patient;

- Individual choice and adaptation of the drug administration form that is best suited to the patient;

- Individualized dosage adapted continuously based on daily experience by both doctor and patient;

- Individualized information to provide optimal patient motivation;

- Individual therapeutic monitoring that generates evidence for the treatment, which can also be used as a supplement to the data from clinical trials;

- Individual support that reinforces daily patient treatment, for example, through compliance systems;

- Individualized distribution, reimbursement and marketing systems. 
Thus, the personalized medicine of the future will include individualized access to the entire course of treatment, thereby optimizing the treatment of the individual, which is what I consider to be truly individualized medicine.

\section{"...pharmacogenetics and \\ pharmacogenomics are simply one of many tools that can be used in the attempt to realize individualized medicine in healthcare systems in the future."}

Obviously, there will be neither the research nor the economic resources available to optimize all of the factors listed above within a given pharmacotherapeutic regime. Therefore, we need clarification of which of the above factors will provide the most individual medicine for our resources; or put in socio-economic terms, how do we get the most individual medicine for the money?
Finally, I need to stress that it is in no way my intention to declare that pharmacogenomics is dead. However, I find it relevant to put pharmacogenomics into a more realistic and holistic perspective for treating our patients. In this perspective, pharmacogenetics and pharmacogenomics are simply one of many tools that can be used in the attempt to realize individualized medicine in healthcare systems in the future.

\section{Financial \& competing interests disclosure}

The author has no relevant affiliations or financial involvement with any organization or entity with a financial interest in or financial conflict with the subject matter or materials discussed in the manuscript. This includes employment, consultancies, honoraria, stock ownership or options, expert testimony, grants or patents received or pending, or royalties.

No writing assistance was utilized in the production of this manuscript.

\section{Bibliography}

1 Seige M: Klinische erfahrungen mit neuronal. Dtsch. Med. Wochenschr. 38, 1828-1830 (1912).

2 Roses AD: Pharmacogenetics and future drug development and delivery. Lancet 355, 1358-1361 (2000).

3 Roses AD: Pharmacogenetics and the practice

of medicine. Nature 405(6788), 857-865 (2000).
4 Sadee W, Dai Z: Pharmacogenetics/ genomics and personalized medicine. Hum. Mol. Genet. 14(Suppl. 2), R207-R214 (2005).

5 Kollek R, van Aken J, Feuerstein G, Schmedders M: Pharmacogenetics, adverse drug reactions and public health. Community Genet. 9(1), 50-54 (2006).

6 Schmedders M, van Aken J, Feuerstein G, Kollek R: Individualized pharmacogenetic therapy: a critical analysis. Community Genet. 6(2), 114-119 (2003).
7 Møldrup C: The prospects and bioethical dimensions of expanding the meaning of pharmacogenomics to compass individualized pharmacotherapy. Per. Med. 2(2), 168-169 (2005) (Abstract).

\section{Website}

101 Pubmed www.ncbi.nlm.nih.gov/pubmed/ 